\title{
Epiphyseal dysplasia of the femoral head, mild vertebral abnormality, myopia, and sensorineural deafness: report of a pedigree with autosomal dominant inheritance
}

\author{
K D MACDERMOT*, S C ROTH $\dagger$, C HALL $\neq$, AND R M WINTER* \\ From * The Kennedy Galton Centre, Harperbury Hospital, and Clinical Research Centre, Watford Road, \\ Harrow; †Guy’s Hospital, London SE1 9RT; and $¥$ The Hospitals for Sick Children, Great Ormond Street \\ London WCIN $3 J H$.
}

SUMmARY A family is presented with short stature, femoral epiphyseal dysplasia, mild vertebral changes, and sensorineural deafness inherited as an autosomal dominant trait. Myopia and retinal detachment presenting in adult life were also present in some affected members. Whe suggest that this disorder may be a distinct entity within the spondyloepiphyseal dysplasia grow of disorders.

The group of conditions classified as multiple epiphyseal dysplasia (MED) and spondyloepiphyseal dysplasia (SED) present with characteristic radiological changes of the epiphyses of the long bones and vertebrae. Associated features, in particular cleft palate, myopia, retinal abnormality, dysmorphic facies, and deafness, have been described singly or in combination in patients with spondyloepiphyseal dysplasia congenita (SEDC). ${ }^{1-3}$ We present a family with an autosomal dominant condition that clearly falls into this group of disorders, but appears to show a previously undescribed combination of features segregating together with minimal variability.

\section{Case reports}

CASE 1

The proband (IV.11, fig 1) was referred because of undiagnosed familial short stature at the age of two and a half years. Pregnancy, delivery, and developmental milestones were unremarkable. The birth length is not known. Her height and weight were recorded as being on the 10th centile at the age of one year and the hip joint was found to be stable at that age. At 18 months of age, a waddling gait was noticed with hip and knee pain which presented as discomfort on movement. An episode was described when the child was unable to get up from kneeling

Received for publication 12 February 1986

Revised version accepted for publication 14 August 1986. position because of inability to straighten her leg. In addition, surgical boots with inserts were needed bscause of abnormally shaped heels. The height a tod weight were just below the 3 rd centile at 18 mont of age. Unilateral hearing loss was discovered of routine testing at two years of age.

On examination at the age of two and $\overrightarrow{\vec{G}}$ half years she had short stature with a short neck and mild shortening of the spine. Lumbar lordose prominent heels, and bilateral clinodactyly were present. The face was triangular, with a protruding forehead and bluish sclerae. There was no apparegुt hypertelorism, malar hypoplasia (fig 2), or cleft. palate. When she was reviewed at the age of three and a half years, her symptoms and clinical findings

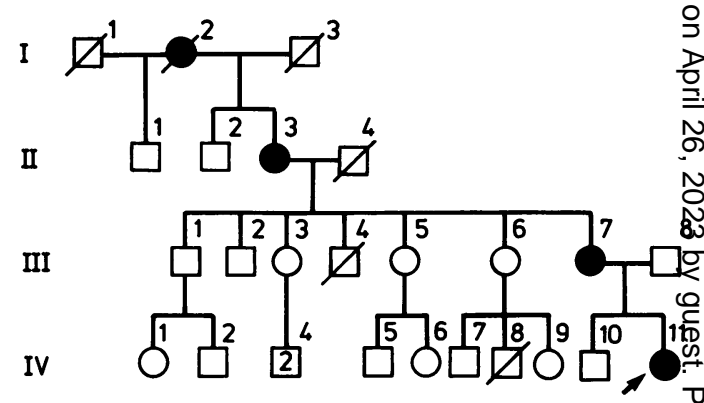

FIG 1 Pedigree illustrating autosomal dominant transmision of epiphyseal dysplasia of the femoral head, mild vertebral abnormality, myopia, and sensorineural deafness. 


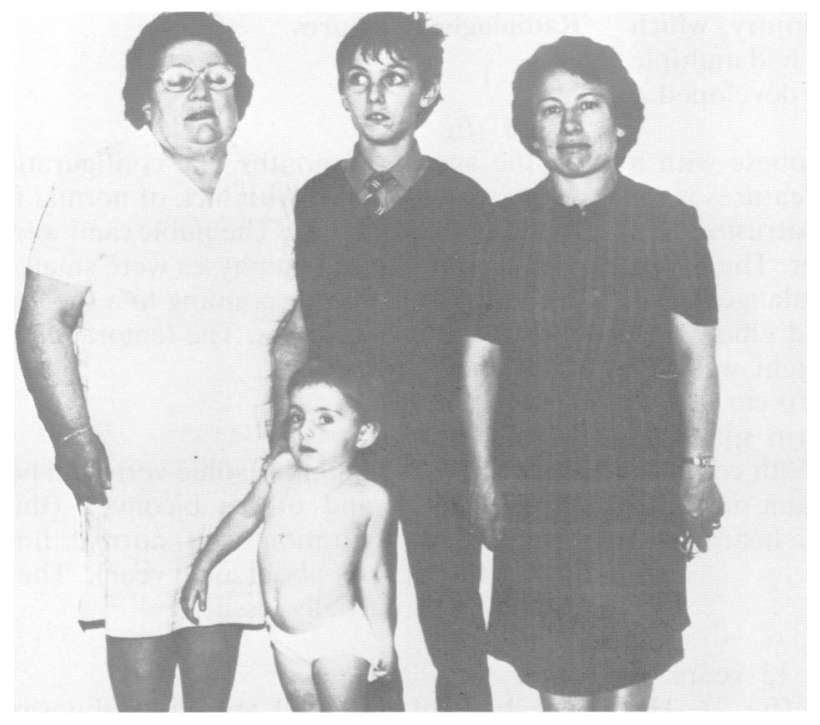

FIG 2 Family photograph showing the proband aged three and a half years (lower centre), proband's mother (right), maternal grandmother (left), and unaffected sib (centre). Normal facial appearance with no evidence of malar hypoplasia.

were unchanged, except that her sclerae were white. There was no joint laxity or stiffness and no history of fractures. The hips were stable. Head circumference was $50.3 \mathrm{~cm}$ (50th centile), weight $12.2 \mathrm{~kg}$ (3rd centile), height $84 \mathrm{~cm}(<3$ rd centile), upper segment $50 \mathrm{~cm}$, lower segment $34 \mathrm{~cm}$ (US/LS ratio 1.47), and arm span $88 \mathrm{~cm}$. Developmental testing showed normal gross and fine motor function, visual spatial performance, and social behaviour. Language comprehension and picture recognition was very good, but pronunciation was poor.

Routine haematological and biochemical parameters including thyroid function test were normal. Two dimensional electrophoresis of urinary glycosaminoglycans showed a normal pattern. Hearing tests were compatible with unilateral sensorineural deafness up to $50 \mathrm{db}$. Ophthalmological examination showed no abnormality of the disc, macula, or retinae and the ocular media were clear. It was not possible to measure visual acuity because of the age of the child, but after dilatation there was no refractive error.

CASE 2 (III.7)

The mother of the proband is aged 36 years and has short stature, deafness, and myopia. The hearing deficit was noted at the age of two years. Her speech is indistinct with a nasal quality. Waddling gait with intoeing was present from an early age, when she also experienced pain in the hips and knees. Subsequently, in her second and third decades, referred pain from the hip joint and cervical spine was her major symptom. Myopia was present from early teens. Her general health is good and she is of average intelligence.

On examination she had a short neck and mild shortening of the spine, disproportionate to the limbs. Her face showed no dysmorphic features and the hands and feet were unremarkable. Mild lumbar lordosis was present. The height was $133 \mathrm{~cm}(<<$ 3 rd centile), weight $46.7 \mathrm{~kg}$, lower segment $64 \mathrm{~cm}$, upper segment $69 \mathrm{~cm}, \mathrm{US} / \mathrm{LS}$ ratio 1.07 , arm span $145 \mathrm{~cm}$, and head circumference $54 \mathrm{~cm}$ (50th centile). A prominent bony protrusion at the occiput was present. She also had bilateral pes planus without a prominent heel and no clinodactyly.

On ophthalmological examination the vision was $6 / 6$ in each eye corrected. The anterior chambers were normal. Following dilatation, negligible peripheral cortical lens opacities were seen in the right eye but they were not thought to represent an early cataract. The retina and vitreous of each eye was normal. Chromosome analysis showed a normal female karyotype.

CASE 3 (II.3)

The maternal grandmother of the proband, aged 65 years, has short stature, deafness, a history of retinal detachment, and has had bilateral hip joint replacement. She has had a waddling, intoeing gait from childhood, but deafness was only noticed in the third decade. Hip and knee pain was present for many years and bilateral hip replacement was indicated in the fourth decade. She is blind in her right eye. The 
blindness is attributed to a childhood injury, which she does not recall. At the age of 40 she had multiple retinal detachments and subsequently developed a cataract in her left eye.

On examination she was short and obese with a very short neck and trunk. Her facial features were unremarkable. A palpable occipital protrusion was present, resembling that of her daughter. The hands showed marked osteoarthritic interphalangeal and metacarpophalangeal enlargements and elbow and finger extension was reduced. Her height was 137 $\mathrm{cm}(<<3$ rd centile), upper segment $70 \mathrm{~cm}$, lower segment $67 \mathrm{~cm}$, US/LS ratio $=1.04$, arm span 146 $\mathrm{cm}$, and head circumference $54.5 \mathrm{~cm}$ (50th centile). Audiometry showed bilateral reduction in both bone and air conduction. She has worn a hearing aid for the last 20 years.

\section{OTHER FAMILY MEMBERS}

The proband's brother (IV.10), aged 12 years, is asymptomatic and of normal stature (fig 2). His height is $141 \mathrm{~cm}$ (25th centile), lower segment 64 $\mathrm{cm}$, upper segment $77 \mathrm{~cm}$, US/LS ratio $1 \cdot 2$, arm span $138 \mathrm{~cm}$, and head circumference $55.5 \mathrm{~cm}(50 \mathrm{th}$ to 90th centile). His hearing is normal and radiological appearance of his hip joint is normal. Family members III.1, 2, 3, 4, 5, and 6 were reported to be asymptomatic, with adult heights of 150 to $160 \mathrm{~cm}$. All their offspring are normal. Subjects II.1 and 2 are still alive, with no evidence of the disorder. I.2 was reported as being short with waddling gait, with perhaps mild deafness, but no history of eye problems.

\section{Radiological features}

CASE 1

Pelvis (fig 3)

At the age of 20 months, the configuration of ili $\overline{\alpha c}$ wings was abnormal with lack of normal flaring and horizontal acetabulae. The pubic rami were ossifieê. The capital femoral epiphyses were small, irregula $\overrightarrow{5}$, and fragmented corresponding to a skeletal matura tion of about six months. The femoral necks showed some medial beaking.

\section{Spine (fig 4)}

At the age of 20 months, some vertebral bodies were 'pear shaped' and others biconvex (this oval or biconvex configuration is a normal finding ond under the age of about one year). The odontoind process was partially ossified.

\section{Skull}

The base of the skull appeared abnormally ste with relative deepening of the middle cranial foss giving rise to an increase in the angle between the floor of the anterior fossa and posterior clivus. wormian bones were present.

\section{Upper and lower limbs}

The long bones were of normal proportions an normally modelled. Apart from the capital femoigl epiphyses, the configuration of epiphyses amd metaphyses was normal. Inferior calcaneal spugs were present bilaterally (fig 5). The bone maturotion at the carpal bones appeared normal at the age

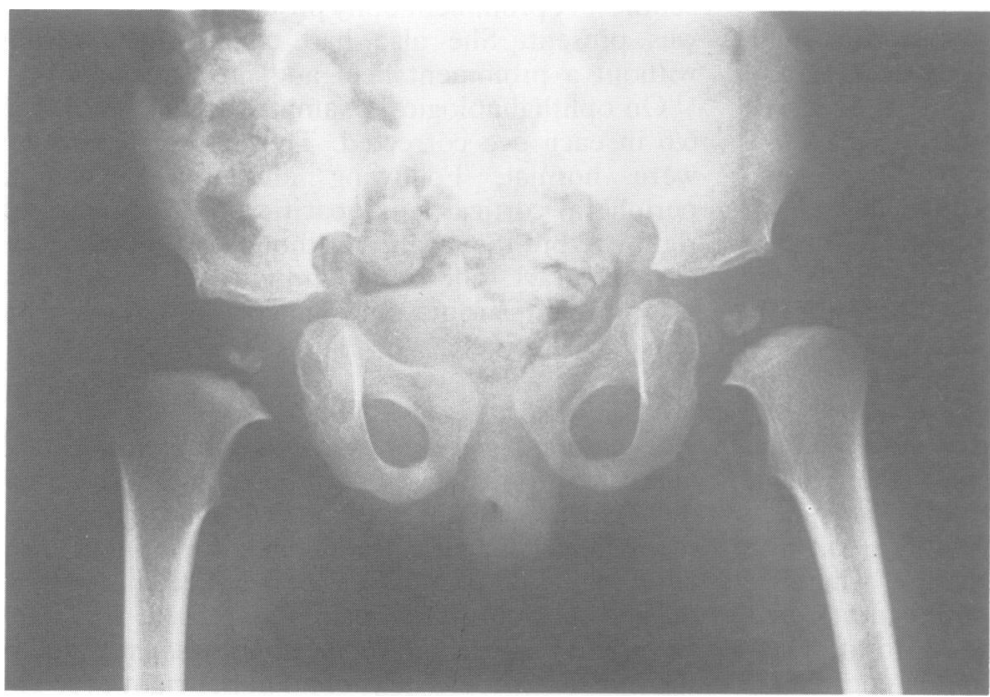

FIG 3 Case 1. AP pelvis at the a of 20 months showing abnormal configuration of iliac wings and 0 horizontal acetabulae. The capitat femoral epiphyses are small and $\mathrm{N}$ irregular with delayed bone maturation. 


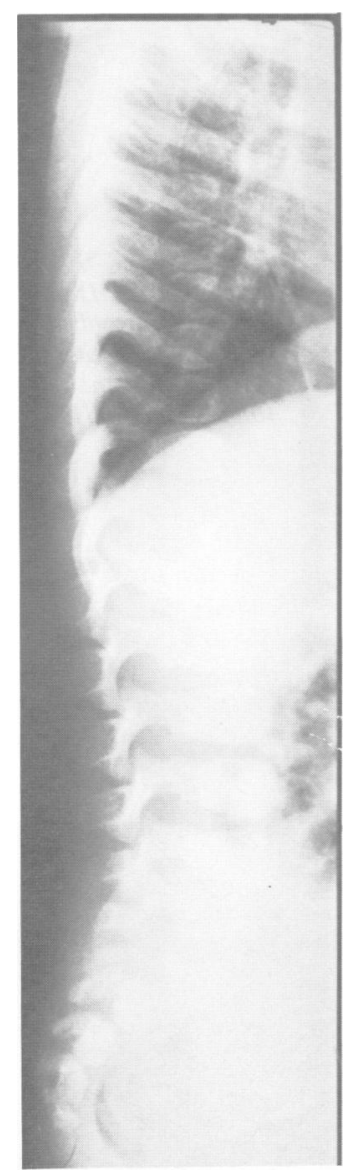

FIG 4 Case 1. Lateral spine at the age of 20 months. Biconvex vertebrae are present in the dorsal region and pear shaped vertebrae in the lumbar region. of 20 months. Clinodactyly and short middle phalanges of the index fingers were present bilaterally (fig 6).

\section{CASE 2}

\section{Pelvis}

The radiological appearance at the age of 27 years showed symmetrical changes with abnormal iliac wing flaring and reduced cephalocaudal height with constriction inferiorly and sloping acetabular roofs. The heads of the femora were flattened and incompletely covered, with subarticular cyst formation and loss of joint space indicating premature osteoarthritis (fig 7). A radiograph of the left hip taken at the age of 12 years showed a small, flat, irregular capital femoral epiphysis with a normal femoral neck (fig 8).

\section{Spine}

Mild flattening with some irregularity of vertebral end plates was present. The pedicles were short with a reduction in the AP diameter of the spinal canal (fig 9).

\section{Upper and lower limbs}

These showed no apparent shortening, normal bone modelling, and no evidence of epiphyseal dysplasia, apart from the capital femoral ephiphyses.

\section{Skull}

The skull and odontoid peg appeared normal.

CASE 3

\section{Pelvis (fig 10)}

The radiological appearance before surgery showed reduced cephalocaudal height of the iliac wings with some constriction inferiorly above the acetabular
FIG 5 Case 1. Lateral left ankle. Calcaneal spur. 


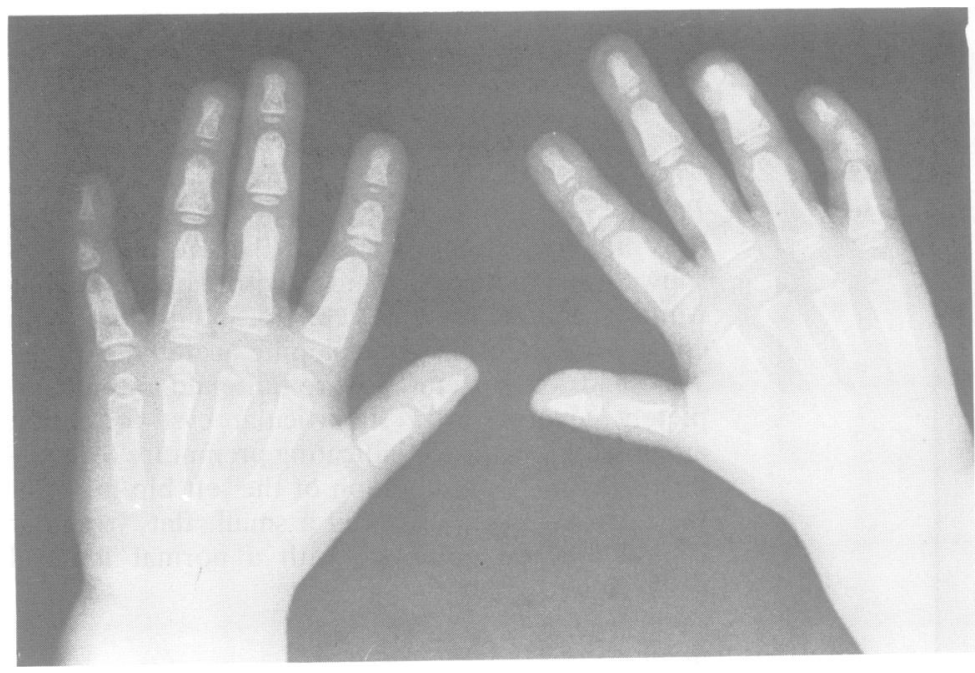

FIG 6 Case 1. Hands at the age of $20 \overrightarrow{\vec{P}}$ months. Normal carpal bone maturation, bilateral clinodactyly, an short middle phalanges of the index $\overrightarrow{3}$ fingers bilaterally.

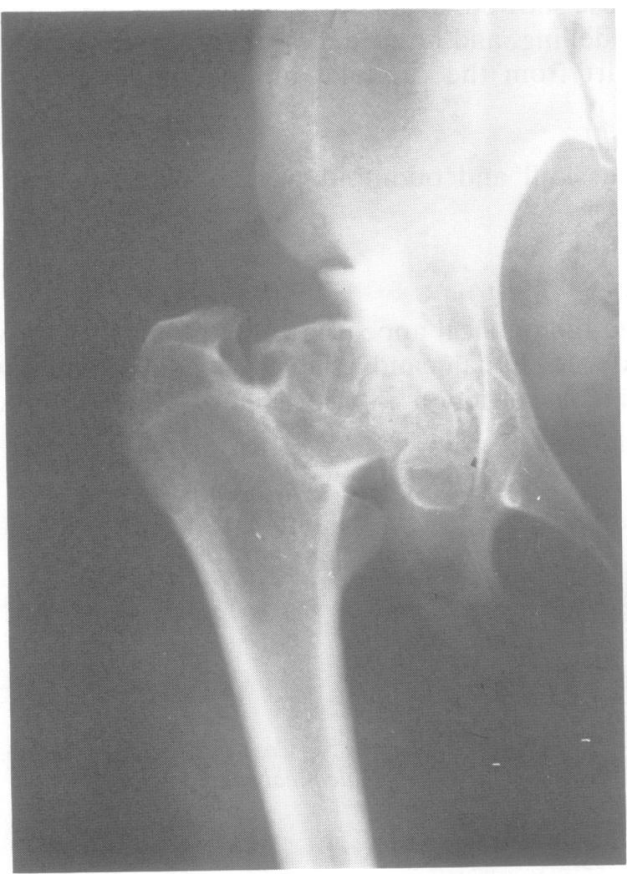

FIG 7 Case 2. AP right hip aged 27 years. Abnormal iliac wing flaring and sloping acetabular roofs. The head of the femur is flattened and incompletely covered, with subarticular cyst formation indicating premature osteoarthritis.

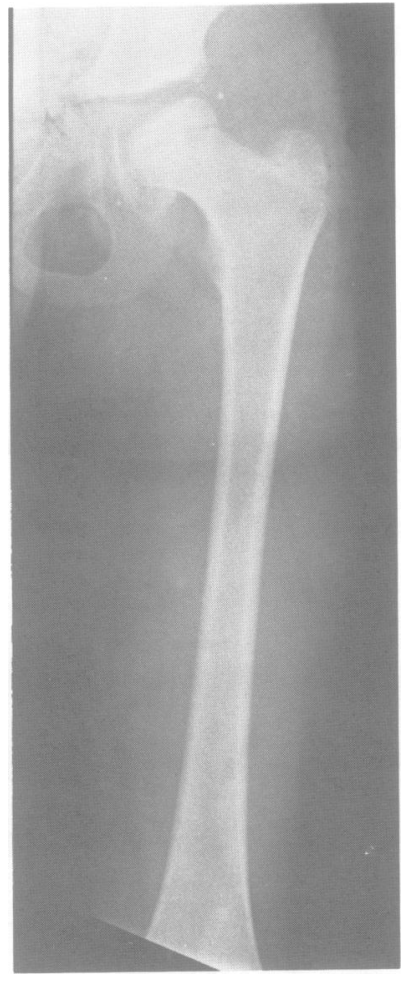

$\stackrel{+}{+}$

$\vec{\circ}$

요

으

○

웅

$\overrightarrow{0}$ 
roofs. The acetabula were sloping, with sclerotic changes secondary to osteoarthritis. The femoral heads were flattened and incompletely covered and the femoral necks were short. There was complete

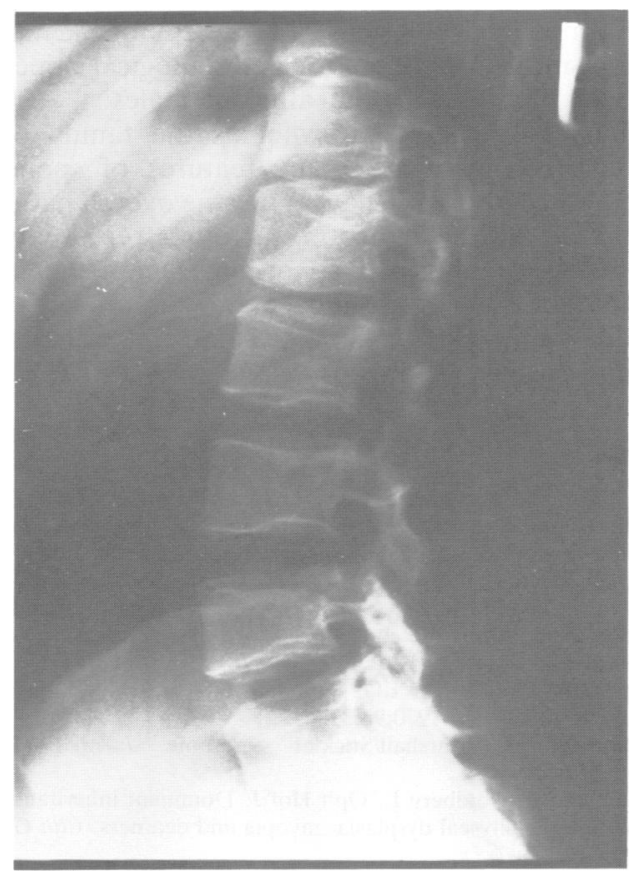

FIG 9 Case 2. Lateral spine. Vertebral end plate irregularity and short pedicles. loss of joint spaces. Recent radiographs showed bilateral hip prostheses.

\section{Spine}

Some platyspondyly and reduced intervertebral spaces were present. Thoracic scoliosis and widespread spondylotic changes were apparent.

\section{Upper and lower limbs}

There was no apparent shortening of long bones and no evidence of epiphyseal dysplasia.

\section{Discussion}

The differential diagnoses in this family included conditions where skeletal abnormalities are associated with deafness and ocular abnormality. The skeletal abnormalities in our family consisted mainly of severe capital femoral epiphyseal dysplasia and mild flattening of vertebral bodies. The pattern of femoral head dysplasia with some vertebral involvement and extraskeletal features resembles spondyloepiphyseal dysplasia congenita (SEDC). ${ }^{3}$ In their survey of 29 patients Spranger and Langer ${ }^{3}$ reported variability of epiphyseal and metaphyseal involvement between different persons and different sites of the skeleton in the same person. The appearance of a short neck, unusual configuration of the pelvis, and dysplasia of the capital femoral epiphyses present in our family have been reported in surveys of cases with SEDC. ${ }^{1-3}$ However, the epiphyseal dysplasia in our family is mainly limited to the hip joint, vertebral changes are mild, and the disorder 'breeds true', suggesting a distinct entity within the

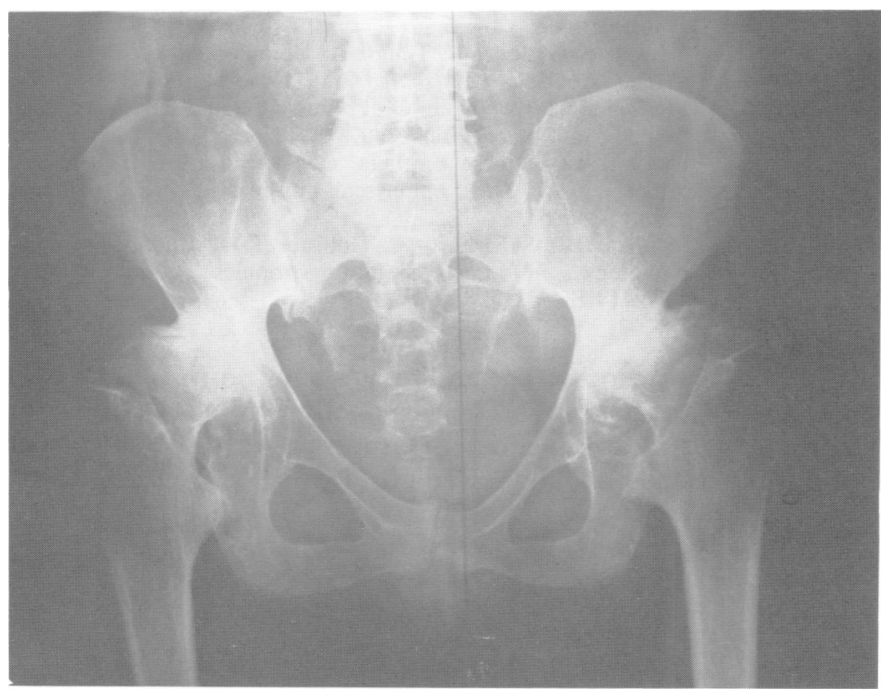

FIG 10 Case 3. AP pelvis. Reduced cephalocaudal height of iliac wings. The acetabula are sloping with sclerotic changes secondary to osteoarthritis. The femoral heads are flattened and incompletely covered. The femoral necks are short. 
group of spondyloepiphyseal dysplasias present at birth.

Our family manifests sensorineural deafness, myopia with retinal detachment, and epiphyseal dysplasia of the hip, all of which are characteristically seen in Marshall/Stickler ${ }^{4}$ syndrome. Dysmorphic facial features, usually present in Marshall/Stickler syndrome, consist mainly of malar hypoplasia, a depressed nasal bridge, and cleft palate. However, in our family the normal facies and marked short stature are not consistent with this diagnosis. Beighton et $a l^{5}$ reported a family with multiple epiphyseal dysplasia, myopia, and deafness, also associated with malar hypoplasia. In addition, retinal thinning and crenated cataracts were found in affected members in this family. Detailed ophthalmological examination showed absence of these findings in affected members of our family. The proband's grandmother (case 3) developed unilateral senile cataract at the age of 60 years. Myopia and retinal detachment were important additional clinical findings in eight of 14 patients with SEDC originally described by Spranger and Langer. ${ }^{3}$ Deafness was described in association with SEDC in three out of 12 patients studied by WynneDavies and Hall, ${ }^{2}$ and Fraser et al ${ }^{1}$ found four out of five patients with deafness in their study of SEDC associated with severe visual handicap. Deafness was not included among the symptoms of SEDC by Spranger and Langer.

Perthes' disease and its variant, dysplasia epiphysealis capitis femoris, ${ }^{6}$ also presents with early childhood hip discomfort, but the evolution of usually unilateral radiological changes is clearly different and when bilateral it is always asymmetrical. Both of these disorders are usually sporadic, but familial cases have been reported. ${ }^{7}$

Namaqualand hip dysplasia ${ }^{8}$ segregates as an autosomal dominant trait and the skeletal changes of early fragmentation and flattening of the capital femoral epiphyses are very similar to those in our family. Degenerative arthropathy subsequently develops, necessitating hip replacement. However, the vertebral changes are not as marked as in our family and the onset is later in childhood. Deafness and eye pathology are not features of Namaqualand hip dysplasia.

Upington disease ${ }^{9}$ consists of Perthes'-like hip changes associated with ecchondromata of peripheral joints, which are not present in our family.
In the syndrome described by Pfeiffer et al, three brothers with epiphyseal dysplasia limited to the femoral head, normal vertebrae, severe myopias and perceptive hearing loss were reported. The parents of these children were related and aute somal recessive inheritance is therefore likely. Th: family most resembles our cases except for the mod of transmission and a normal radiological appeast ance of the spine in the affected males.

Thus, it appears that whereas the family pre sented here manifests many features of sponds loepiphyseal dysplasia congenita, the overall patte of abnormalities segregating together represents is previously undescribed condition. Detailed hist $\odot-$ pathological $^{11} 12$ and molecular studies will be needed for the precise subdivision of this heters geneous group of disorders.

\section{References}

I Fraser GR, Friedmann AI, Maroteaux P, Glen-Bott Af, Mittwoch U. Dysplasia spondiloepiphysaria congenita and lated generalised skeletal dysplasias among children with sevene visual handicaps. Arch Dis Child 1969;44:490-8.

2 Wynne-Davies R, Hall C. Two clinical variants of spondy $\overrightarrow{8}$ epiphysial dysplasia congenita. J Bone Joint Surg (BK) 1982;64:435-41.

3 Spranger JW, Langer LO. Spondyloepiphyseal dysplasia cengenita. Radiology 1970;94:313-22.

4 Baraitser M. Marshall/Stickler syndrome. J Med Ge官 1982;19:139-40.

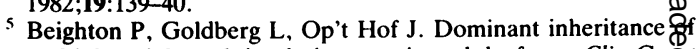
multiple epiphyseal dysplasia, myopia and deafness. Clin Geât

- Meyer J. Dysplasia epiphysealis capitis femoris. Acta Orth

7 Wcand 1964;34:183-97. Am J Dis Child 1963;106:131-4.

${ }^{8}$ Beighton P, Christy G, Learmonth ID. Namaqualand hip dysplasia: an autosomal dominant entity. Am J Med Ge

9 1984;19:161-9. dyschondroplasia. S Afr Med J 1971;45:994-1000.

10 Pfeiffer RA, Jünemann G, Polster J, Bauer H. Epiphysßal dysplasia of the femoral head, severe myopia and perceptije hearing loss in three brothers. Clin Genet 1973;4:141-4.

1 Spranger J. The epiphyseal dysplasias. Clin Orth

12 Rimoin DL, Silberberg R, Hollister DW. Chondro-osse更s
1974:46-60. pathology in the chondrodystrophies. Clin Orthop 1976;114:137-52.

Correspondence and requests for reprints to $\mathrm{Dr}$ MacDermot, The Kennedy-Galton Centre for Clinical Genetics, Harperbury Hospital, Harper Lane, Shenley, Radlett, Herts WD7 9HQ. 